\title{
Unusual Presentation of Renal Cell Carcinoma in Crossed Ectopic Kidney
}

\author{
Mohammad Reza Nowroozi ${ }^{1}$; Hamidreza Ghorbani ${ }^{1}$; Erfan Amini ${ }^{1}$; Amir Arbab ${ }^{1}$; Alireza \\ Ghadian ${ }^{2, *}$ \\ ${ }^{1}$ Uro-Oncology Research Center, Tehran University of Medical Sciences, Tehran, IR Iran \\ ${ }^{2}$ Nephrology and Urology Research Center, Baqiyatallah University of Medical Sciences, Tehran, IR Iran \\ *Corresponding author: Alireza Ghadian, Nephrology and Urology Research Center, Baqiyatallah University of Medical Sciences, Tehran, IR Iran. Tel: +98-2181262073, \\ E-mail: P_Ghadian@yahoo.com
}

Received: January 11, 2015; Accepted: February 14, 2015

\begin{abstract}
Introduction: Crossed renal ectopia is a rare anomaly and ninety percent of crossed ectopic kidneys are fused to their ipsilateral mate. Based on autopsy findings, the incidence has been estimated to be one in 2000 individuals.

Case Presentation: We hereby report on a 53-year-old woman with two episodes of painless gross hematuria. Imaging revealed left side fused crossed renal ectopia and filling defect within the pyelocaliceal of crossed kidney.

Conclusions: The patient underwent surgery applying a midline incision. The left kidney showed a lump pattern embedded in lower pole of the right kidney. Left sided nephrectomy was performed while temporary right renal artery was clamped temporarily. Histopathological evaluation revealed clear cell carcinoma with severe nuclear atypia (Fuhrman grade 4/4). However, local recurrence was not detected during the 18-month follow up after surgery.
\end{abstract}

Keywords: Renal Cell Carcinoma; Crossed Renal Ectopia; Malignancy

\section{Introduction}

Crossed renal ectopia is a rare anomaly and ninety percent of crossed ectopic kidneys are fused to their ipsilateral mate. Based on autopsy findings, the incidence has been estimated to be one in 2000 individuals. Unilaterally fused kidney with inferior ectopia is the most common variety, whereas fusion with superior ectopia is the least common (1). The association of a malignant tumor with this anomaly is extremely rare $(2,3)$. Only four tumors in crossed kidneys have been reported and histopathological evaluations have confirmed renal cell carcinoma in all aforesaid tumors (1).

\section{Case Presentation}

A 53-year-old woman was admitted to our department with two episodes of painless gross hematuria during the last three months. She had no history of systemic diseases, constitutional symptoms and familial history of urological disorders. Physical exam revealed a palpable ill-defined mass in the right upper quadrant with extension to the umbilical region. Serum creatinine was $1.4 \mathrm{mg} / \mathrm{dL}$, and urine analysis revealed microscopic hematuria (20-25 RBC / HPF). Urine cytology was negative for malignancy. Intravenous Urography (IVU) revealed left side fused crossed renal ectopia and filling defect within the pelvis of the crossed kidney (Figure 1 A). A filling defect was evident in the left crossed fused kidney in retrograde pyelography (Figure $1 \mathrm{~A}$ ). Computerized-tomography (CT) scan confirmed the presence of left crossed renal ectopia associated with an enhancing mass, arising from the pelvis and pyelocaliceal system of the crossed kidney (Figure 1 C, 1D). Cystoscopy showed no abnormality within the bladder. Ureteral orifices were normal. Chest X-ray and liver function tests were normal. The patient underwent surgery applying a midline incision. Left kidney showed a lump pattern, embedded in the lower pole of the right kidney. Both pelvises of kidneys were located anteriorly. Left renal pelvis and parapelvis tissue were firm.

The main renal arteries were dissected thoroughly. Interestingly, when the left main renal artery was clamped temporarily, both kidneys became partially ischemic, whereas clamping the right renal artery was associated with right renal ischemia with no effect on the crossed left sided kidney. During the right renal artery clamping, left nephrectomy was performed. All bleeding sites were ligated with absorbable sutures. Left ureter was resected near the bladder. Frozen section from margins indicated no evidence of tumor involvement. No palpable regional lymph node was detected. Post-operative course was uneventful. Macro-

Copyright (C) 2015, Nephrology and Urology Research Center. This is an open-access article distributed under the terms of the Creative Commons Attribution-NonCommercial 4.0 International License (http://creativecommons.org/licenses/by-nc/4.0/) which permits copy and redistribute the material just in noncommercial usages, provided the original work is properly cited. 
scopic evaluation of the specimen revealed a $9 \times 7 \times 5.5$ $\mathrm{cm}$ kidney with a well-defined mass, which had variegated cut surfaces measuring $8 \times 5 \times 4 \mathrm{~cm}$ with extension into the proximal ureter.

Microscopic evaluation showed a malignant renal tumor with solid and alveolar patterns, clear cell morphology and severe nuclear atypia (Fuhrman grade $4 / 4$ ). Immunohistochemistry evaluation showed negative reaction for CK7, CK20, CK5 and CK6, and high nuclear weight cy- tokeratin in favor of clear cell type renal cell carcinoma. Renal sinus and pyelocaliceal system were invaded by tumors. However, the perirenal fat was tumor free. No vascular invasion was noted and all surgical margins were tumor free. Magnetic Resonance Imaging (MRI) and ultrasonography was applied on a regular basis to detect any evidence of local recurrence. Nevertheless, local recurrence was not detected during the 18-month follow up after surgery (Figure 2).
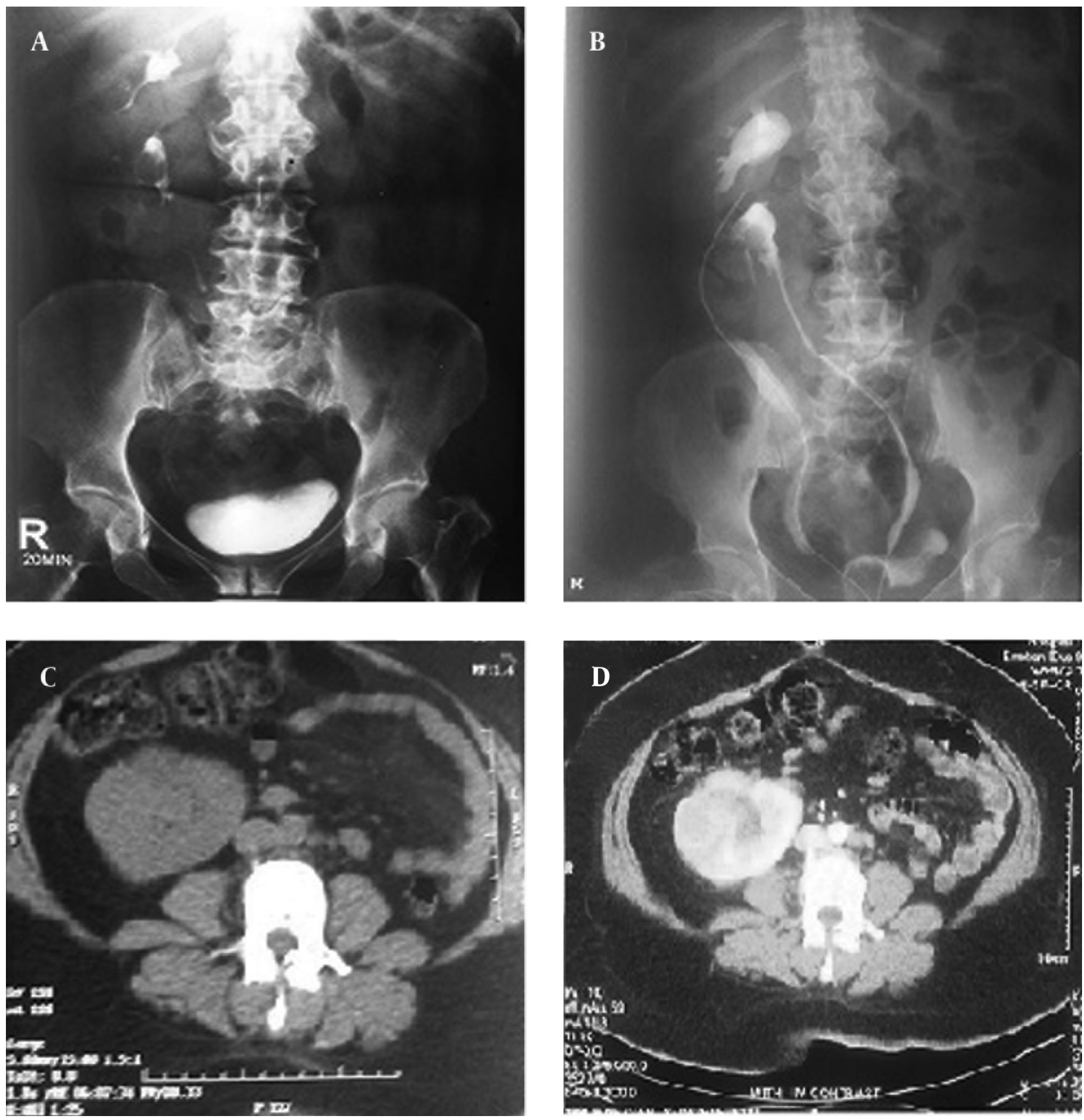

Figure 1. IUV and CT of Patient 

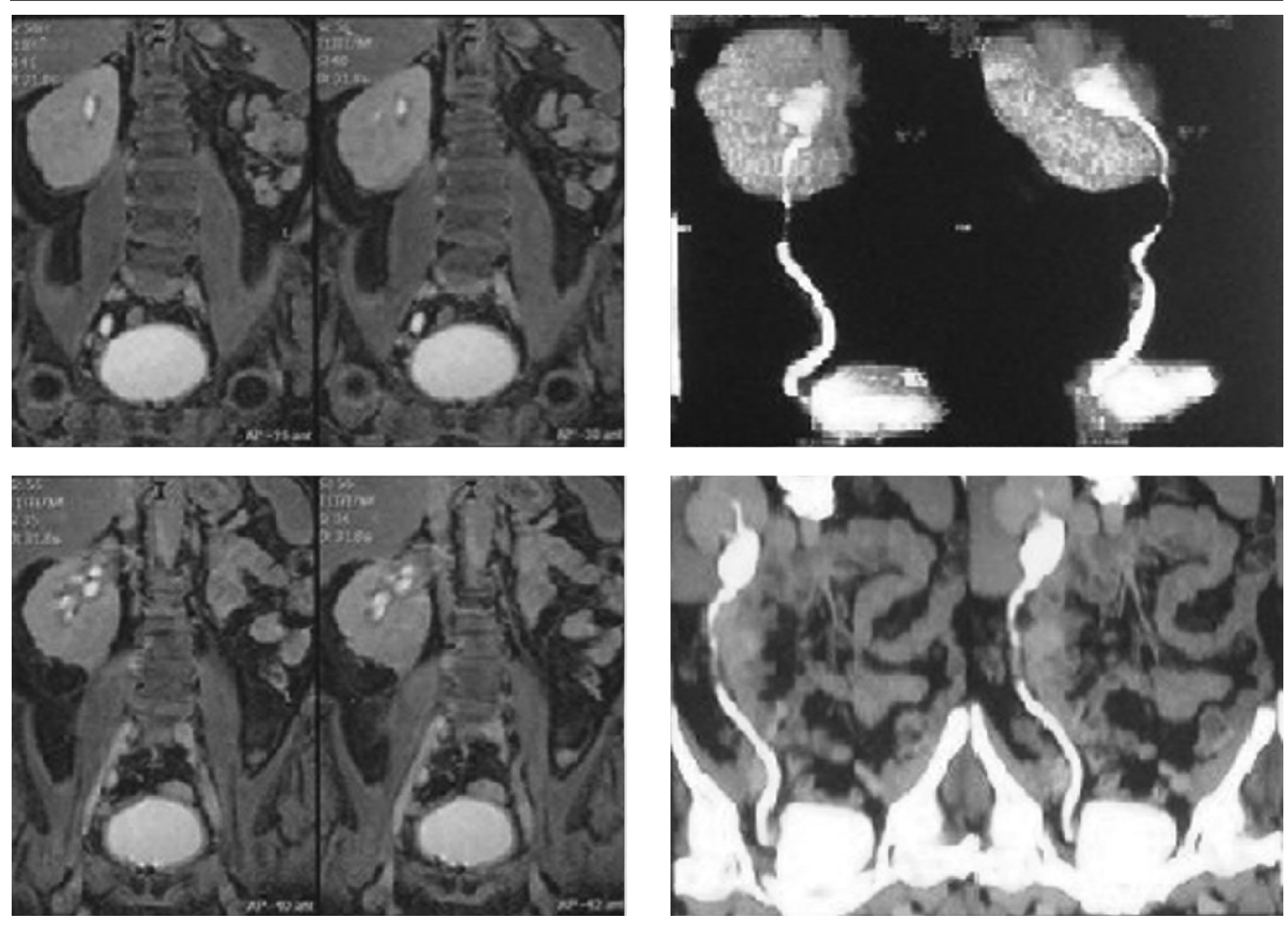

Figure 2. MRI After 18 Months

\section{Discussion}

Crossed renal ectopia is an uncommon congenital anomaly and in most cases usually presents fusion of both kidneys. The incidence has been estimated to be one in 2000 live births (1). There is a mild male predominance and left to right cross over occurs more frequently (1). The association of malignant tumor with such anomalies is extremely rare $(2,3)$. Computerize tomography scan is the modality of choice in detecting tumor in fusion anomaly. The surgical approach for ectopic and especially fused kidneys merits caution because of uncertain anatomy and vascularization (4).

Although a vascular study (i.e. angiography) was not performed for the present case, preoperative vascular studies are of utmost importance and ensure proper interruption of the blood supply prior to resection. A good vascular map before surgery can avoid significant hemorrhage during operation and decreases operation time. We recommend trans-peritoneal nephrectomy for patients with crossed fused ectopia, which is associated with optimal access to main vessels.

\subsection{Conclusions}

Kidneys with crossed fused ectopia rarely involved a renal mass. Renal cell carcinoma is the most frequent tumor type associated with fusion anomalies. Nephrectomy is necessary in such cases and vascular mapping prior to surgery may help avoid significant hemorrhage during surgery and decreases ischemia of the remaining kidney.

\section{Authors' Contributions}

Dr. Mohammad Reza Nowroozi, Dr. Hamidreza Ghorbani and Dr. Amir Arbab carried out the operations, Dr. Erfan Amini collected the data and Dr. Alireza Ghadian contributed to analysis and writing of the manuscript.

\section{References}

1. Shapiro E, Bauer SB, Chow JS. Anomalies of the Upper Urinary Tract. In: Walsh PC, Retic AB, Vaughan DE, Wein AJ editors. Campbell s urology.. Philadelphia: WB saunders; 2012. pp. 3140-4.

2. Bolton DM, Bowsher WG, Costello AJ. Renal cell carcinoma in both moieties of crossed fused ectopia. Aust $N$ Z J Surg. 1993;63(8):662-3.

3. Sugita S, Kawashima H, Nakatani T, Yoshimura R, Wada S, Sugimura $\mathrm{K}$, et al. Renal cell carcinoma in an L-shaped kidney. Int J Urol.2000;7(6):236-8.

4. Stimac G, Dimanovski J, Ruzic B, Spajic B, Kraus O. Tumors in kidney fusion anomalies--report of five cases and review of the literature. Scand J Urol Nephrol. 2004;38(6):485-9. 